\title{
Original
}

\section{Infrahyoid Myocutaneous Flap Versus Radial Forearm Free Flap in Treating Patients with cT1-2 Tongue Carcinoma}

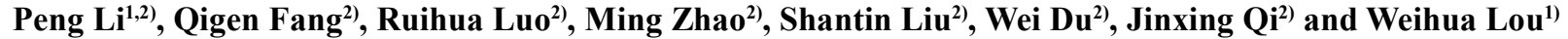 \\ 1) Department of Otorhinolaryngology Head and Neck Surgery, The First Affiliated Hospital of Zhengzhou University, Henan, China \\ ${ }^{2}$ Department of Head Neck and Thyroid Surgery, The Affiliated Tumor Hospital of Zhengzhou University, Henan, China \\ (Accepted for publication, June 23, 2015)
}

\begin{abstract}
Our goal was to compare clinical and functional results of the infrahyoid myocutaneous flap (IMF) with those of the radial forearm free flap (RFFF) for partial tongue reconstruction. Between January 2005 and June 2012, patients who had received an IMF or a RFFF reconstruction for cT1-2 tongue carcinoma were included, their medical records were reviewed, and they were asked to complete the University of Washington Quality of Life (UW-QoL) version 4 questionnaire at least 12 months postoperatively. Compared to patients with a RFFF reconstruction, patients treated with an IMF had significantly shorter operation time $(\mathrm{p}<0.001)$, less hospital cost $(\mathrm{p}<0.001)$ and shorter postoperative stay $(\mathrm{p}<0.001)$. There were no apparent differences in other scales between the two groups. The mean composite score for the two groups was 88.4 (SD: 14.9) and 86.4 (SD: 15.3), respectively, the finding was not significant $(\mathrm{p}=0.784)$. Patients with an IMF reconstruction had significantly better shoulder function, there were no significant differences regarding other domains between the two groups. Compared to RFFF, IMF was a reliable procedure for partial tongue reconstruction.
\end{abstract}

Key words: Quality of life, Infrahyoid myocutaneous flap, Radial forearm flap, Tongue reconstruction

\section{Introduction}

Tongue carcinomas are the most common malignancy in oral cavity $^{1)}$, these lesions severely affect patients' quality of life and pose significant threat to their lives. Generally, complete and extensive resection is a reliable and current method to improve local control of the diseases, and the surgeon also cares about the postoperative functional results such as speech, swallowing and so on. Since the infrahyoid myocutaneous flap (IMF) was first introduced by Wang et al. ${ }^{2}$, it has been widely used in reconstruction of small- and medium-sized defects of the oral cavity $^{3-7)}$. Nowadays, due to advancements of microvascular surgery, free flap, especially radial forearm free flap (RFFF) has been the preferred method for soft tissue reconstruction ${ }^{8)}$. Thus, are there significant differences between the two procedures? Few reports to date are available, therefore, our goal was to compare clinical and functional results of the IMF with those of the RFFF for tongue reconstruction.

\section{Materials and Methods}

The Zhengzhou University institutional research committee

Correspondence to: Dr Weihua Lou, Department of Otorhinolaryngology Head and Neck Surgery, The First Affiliated Hospital of Zhengzhou University, Zhengzhou, Henan, China; Tel: 86 18838081528; Fax: 86 18638588830; Email: lipeng1528@163.com approved our study, and all participants signed an informed consent agreement.

We retrospectively reviewed medical records from January 2005 to June 2012 in the Department of Head and Neck \& Thyroid Surgery, the Affiliated Cancer Hospital of Zhengzhou University. Enrolled patients were required to meet the following criteria: the primary diagnosis was cT1-2 tongue carcinoma according to UICC 2002 classification; no preoperative surgical or radiation treatments; IMF or RFFF was used for tongue reconstruction; no previous history of cancer, and no impairments in communication; follow-up duration longer than 12 months. These patients were divided into two groups: in group A, all patients received an IMF reconstruction; in group $\mathrm{B}$, all patients were treated with a RFFF. The medical records of included patients were reviewed, and furthermore they were asked to complete the University of Washington Quality of Life (UW-QoL) version 4 questionnaire. All these patients underwent primary tumour resection and unilateral selective neck dissection (SND).

The harvest of a RFFF was performed according to previous description $^{8}$. Simultaneous flap elevation and recipient site surgery were performed to shorten the total operation time. The donor site was closed using a split thickness skin graft.

The harvest of an IMF was performed based on previous studies $^{2-7)}$. The IMF was elevated from caudal to cephalad. To 
J.Hard Tissue Biology Vol. 24(3):285 -288, 2015

Table 1. Characteristics of Patients who had Returned the Questionnaire

\begin{tabular}{|c|c|c|c|}
\hline & Group A $(n=18)$ & Group B $(n=38)$ & $\mathrm{p}$ \\
\hline Age (years) & $53.3(45-61)$ & $52.5(33-65)$ & 0.799 \\
\hline \multicolumn{4}{|l|}{ Sex } \\
\hline Male & 12 & 22 & \multirow[t]{2}{*}{0.530} \\
\hline Female & 6 & 16 & \\
\hline \multicolumn{4}{|l|}{ Tumor stage } \\
\hline $\mathrm{T} 1$ & 0 & 4 & \multirow[t]{2}{*}{0.294} \\
\hline $\mathrm{T} 2$ & 18 & 34 & \\
\hline \multicolumn{4}{|l|}{ TNM stage } \\
\hline I & 0 & 4 & \multirow[t]{2}{*}{0.294} \\
\hline II & 18 & 34 & \\
\hline Flap size $\left(\mathrm{cm}^{2}\right)$ & $55(36-108)$ & $60(35-120)$ & 0.873 \\
\hline Flap total survival rate & $94.4 \%$ & $100 \%$ & 0.321 \\
\hline Wound infection & 0 & 0 & - \\
\hline Postoperative radiation & 2 & 5 & 1.000 \\
\hline \multicolumn{4}{|l|}{ Operation time } \\
\hline$\leqq 80$ minutes & 15 & 7 & \multirow[t]{2}{*}{$<0.001$} \\
\hline$>180$ minutes & 3 & 31 & \\
\hline Hospital cost (RMB) & 24,000 & 41,000 & $<0.001$ \\
\hline Postoperative stay (days) & 9.3 & 12.7 & $<0.001$ \\
\hline
\end{tabular}

promise adequate blood supply, the main trunk and anterior branch of the superior thyroid artery were kept with the flap, and the posterior branch was selectively tied. Careful attention should be taken for identifying and preserve all the veins in the direction of the internal jugular vein. The descending branch of the hypoglossal nerve was preserved to improve postoperative function.

The UW-QoL scale consisted of 12 single-question domains that had between 3 and 6 response options, scaled evenly from 0 (worst) to 100 (best). The domains were pain, appearance, activity, recreation, swallowing, chewing, speech, shoulder, taste, saliva, mood and anxiety. The individual domains were scored according to the UW-QoL guidelines.

The chi-square test and student $t$ test were used to assess the general variables, the nonparametric Mann-Whitney test was used to evaluate the UW-QoL scores, and all statistic analyses were performed using SPSS 13.0. A $p<0.05$ was considered significant.

\section{Results}

Finally $56(87.5 \%)$ of 64 patients completed the questionnaire, their detail information was described in Table 1. No neck node metastasis was found pathologically. Compared to patients with a RFFF reconstruction, patients treated with an IMF had significantly shorter operation time $(\mathrm{p}<0.001)$, less hospital cost $(p<0.001)$ and shorter postoperative stay $(p<0.001)$. There were no apparent differences in other scales between the two groups.

As Table 2 presented, the mean composite score for the two groups was 88.4 (SD: 14.9) and 86.4 (SD: 15.3), respectively, the finding was not significant $(\mathrm{p}=0.784)$. Patients in group A had significantly better shoulder function, there were no significant differences regarding other domains between the two groups.

\section{Discussion}

In current study, flap size did not differ significantly between the two groups, but a similar study by Deganello et al. ${ }^{5}$ presented a larger skin paddle surface in patients with RFFFs. The difference could be explained by that we just focused on cT1-2 lesions. Furthermore, the finding supported that the IMF might be suitable for small- and middle-size defects ${ }^{3,4)}$.

We observed that free flap reconstructions required longer operative times. Similar findings have also been reported by Hsing et al. ${ }^{9)}$ and Mallet et al. ${ }^{10)}$. Chepeha et al. ${ }^{11)}$ demonstrated that patients undergoing pedicled flap reconstructions had longer hospital stays than patients receiving free flaps. Similarly, de Bree et al. ${ }^{12)}$ reported that compared with the pedicle flap group, mean hospital stay was significantly shorter in the free flap group (24 vs 28 days, $\mathrm{P}=0.005$ ). However, we found that postoperative hospital stays were significantly longer in the free flap group. Several factors may account for this difference. First, the complication rate of our pedicle flaps was lower than in the aforementioned studies. Furthermore, our definition of duration was different, as we only calculated the length of the postoperative hospital stay. Finally, we only included patients with cT1-2 tongue cancers, early stage lesions had a relatively low incidence of complication, but the 
Peng Li et al.: Infrahyoid Flap Versus Radial Forearm Flap

Table 2. Comparison of Outcomes of Quality of Life between the Two Groups

\begin{tabular}{|c|c|c|c|}
\hline Domains & Group A $(n=18)$ & Group B $(n=38)$ & $\mathrm{p}$ \\
\hline Pain & 94.4(SD: 11.0) & 93.4(SD:11.3) & 0.819 \\
\hline Appearance & 80.6(SD: 20.8) & 76.3(SD: 19.5) & 0.582 \\
\hline Activity & 94.4(SD: 11.0) & 90.8(SD: 12.4) & 0.447 \\
\hline Recreation & 83.3(SD: 17.7) & 86.8(SD: 15.3) & 0.620 \\
\hline Swallowing & 93.3(SD: 13.2) & 92.1(SD: 13.6) & 0.819 \\
\hline Chewing & 94.4(SD: 16.7) & 89.5(SD: 20.9) & 0.529 \\
\hline Speech & 86.7(SD: 15.8) & 84.2(SD: 15.4) & 0.691 \\
\hline Shoulder & 96.7(SD: 10.0) & 84.2(SD: 15.4) & 0.039 \\
\hline Taste & 86.7(SD: 15.8) & 89.0(SD: 14.9) & 0.706 \\
\hline Saliva & $90.0(\mathrm{SD}: 15.0)$ & 87.4(SD: 15.2) & 0.663 \\
\hline Mood & 80.6(SD: 24.3) & 79.0(SD: 22.5) & 0.764 \\
\hline Anxiety & $80.0(\mathrm{SD}: 15.0)$ & 84.2(SD: 15.4) & 0.491 \\
\hline
\end{tabular}

other studies included patients who underwent a second extirpation as well as those whose primary cancer originated from sites other than the oral cavity.

De Bree et al. ${ }^{12)}$ reported that mean surgical costs were significantly higher for patients reconstructed with a free flap instead of a pedicle flap but that in the postoperative period, mean hospital costs were lower. Fattabi et al. ${ }^{13)}$ found that direct surgical fees and total hospital charges were higher for a microvascular reconstruction than a pedicle flap reconstruction. In the present study, we also noted that free flap reconstructions were associated with higher total hospital costs. However, health care systems and economic conditions might differ among these studies. In our cancer center, patients with oral cancers often came from low income family and had no medical insurance, a high hospital cost was a great burden for these patients.

Successful surgical treatments should not only resect the tumor completely but also achieve wonderfully functional rehabilitation, thus, quality of life (QoL) was increasingly becoming an important assessment of outcome in oncological research ${ }^{14)}$. The UW-QoL questionnaire has some advantages: it is multi-factorial, allowing sufficient detail to identify subtle change; it is brief and selfadministered; it could reflect the QoL as indicated by the patient due to allowing no input from the health provider; it provides questions specific to head and neck cancer. The scale has been proven to be charactered by high validity and reliability ${ }^{15)}$.

Good speech and swallowing capacity was the principle of tongue reconstruction. Windfuhr et al. ${ }^{6}$ described maintenance of solid food and swallowing without reclination of the head was possible for all patients with IMF. Similarly, Ouyang et al. ${ }^{7}$ presented their experience of IMF reconstruction and reported all the patients achieved satisfactory function rehabilitation. In current study, we also noted patients in the IMF group had good speech and swallowing capacity, and it was consistent with the outcome of the RFFF group. Furthermore, the finding was confirmed by an objective measurement. Deganello et al. ${ }^{5)}$ found no significant differences were found with regard to verbal intelligibility and diet scores between patients with IMF and RFFF. Joo et al. ${ }^{16)}$ presented postoperative flap volume changes were significantly correlated with swallowing and speech outcomes in patients underwent partial glossectomy. Unfortunately tissue atrophy was inevitable after the denervated flap was transferred, such as RFFF. But in our IMF designation, we preserved the motor innervations of the infrahyoid muscles provided by the ansa cervivalis, it could prevent atrophy of the reconstructed tongue. Therefore, we reputed that long term functional results might be better in patients with IMF.

Patients with an IMF reconstruction had significantly better shoulder function. Possible causes might be the neck dissection and the RFFF harvest. But the effect of SND on shoulder function remained unclear, Kuntz et al. ${ }^{(17)}$ found that the SND group reported near normal shoulder function at both 6 and 12 months. Teymoortash et al. ${ }^{(18)}$ claimed SND of different dimensions and neck levels showed low incidence of surgical complication and acceptable functional and aesthetic results. Therefore, the negative effect on shoulder function of SND was limited. Previous authors had described RFFF had a significant deleterious effect on upper extremity function by a prospective design ${ }^{19}$.

Some shortcomings existed in our research. First, it was retrospective so selection bias could not be avoided. Second, only 56 patients were enrolled in this study; in order to generate more conclusive results a larger group of patients need to be studied.

In summary, compared with the RFFF group, infrahyoid myocutaneous flap reconstruction required shorter surgical time and postoperative hospitals stays, and lower hospital costs, and could achieve good speech and swallowing capacity, and better shoulder function. Infrahyoid myocutaneous flap was a reliable procedure for partial tongue reconstruction.

\section{References}

1. Fang QG, Shi S, Liu FY and Sun CF. Tongue squamous cell 
carcinoma as a possible distinct entity in patients under 40 years old: a distinct entity? Oncol Lett 7: 2099-2102, 2014

2. Wang HS, Shen JW, Ma DB, Wang JD and Tian AL. The infrahyoid myocutaneous flap for reconstruction after resection of head and neck cancer. Cancer 57: 663-668, 1986

3. Zhao YF, Zhang WF and Zhao JH. Reconstruction of intraoral defects after cancer surgery using cervical pedicle flaps. J Oral Maxillofac Surg 59: 1142-1146, 2001

4. Tincani AJ, Negro AD, Araujo PP, Neves Fda S and Martins AS. Head and neck reconstruction using infrahyoid myocutaneous flaps. Sao Paulo Med J 124: 271-274, 2006

Deganello A, Gitti G, Parrinello G, Larotonda G, Meccariello G, Leemans CR and Gallo O. Infrahyoid flap reconstruction of oral cavity and oropharyngeal defects in elderly patients with severe general comorbidities. Head Neck 34: 12991305,2012

6. Windfuhr JP and Remmert S. Infrahyoid myofascial flap for tongue reconstruction. Eur Arch Otorhinolaryngol 263: 1013-1022, 2006

7. Ouyang D, Su X, Chen WC, Chen YF, Men QQ and Yang AK. Anatomical study and modified incision of the infrahyoid myocutaneous flap. Eur Arch Otorhinolaryngol 270: 675-680, 2013

8. Fang QG, Li ZN, Zhang X, Liu FY, Xu ZF and Sun CF. Clinical reliability of radial forearm free flap in repair of buccal defects. World J Surg Oncol 11: 26, 2013

9. Hsing CY, Wong YK, Wang CP, Wang CC, Jiang RS, Chen FJ and Liu SA. Comparison between free flap and pectoralis major pedicled flap for reconstruction in oral cavity cancer patients-a quality of life analysis. Oral Oncol 47: 522-527, 2011

10. Mallet Y, EI Bedoui S, Penel N, Ton Van J, Fournier C and Lefebvre JL. The free vascularized flap and the pectoralis major pedicled flap options: comparative results of reconstruction of the tongue. Oral Oncol 45: 1028-1031,
2009

11. Chepeha DB, Annich G, Pynnonen MA, Beck J, Wolf GT, Teknos TN, Bradford CR, Carroll WR and Esclamado RM. Pectoralis major myocutaneous flap vs revascularized free tissue transfer. Arch Otolaryngol Head Neck Surg 130: 181186, 2004

12. de Bree R, Reith R, Quak JJ, Uyl-de Groot CA, van Agthoven $\mathrm{M}$ and Leemans CR. Free radial forearm flap versus pectoralis major myocutaneous flap reconstruction of oral and oropharyngeal defects: a cost analysis. Clin Otolaryngol 32: 275-282, 2007

13. Fattabi $\mathrm{T}$ and Fernandes R. Value of microvascular surgery in academic oral and maxillofacial surgery. J Oral Maxillofac Surg 71: 189-191, 2013

14. A, Sun KC, DeSilva RN and Smith AC. The assessment of quality of life in patients who have undergone surgery for oral cancer: a preliminary report. J Oral Maxillofac Surg 63: 1606-1612, 2005

15. Fang QG, Shi S, Zhang X, Li ZN, Liu FY and Sun CF. Assessment of the quality of life of patients with oral cancer after pectoralis major myocutaneous flap reconstruction with a focus on speech. J Oral Maxillofac Surg 71: e1-e5, 2013

16. Joo YH, Hwang SH, Park JO, Cho KJ and Kim MS. Functional outcome after partial glossectomy with reconstruction using radial forearm free flap. Auris Nasus Larynx 40: 303-307, 2013

17. Kuntz AL and Weymuller EA. Impact of neck dissection on quality of life. Laryngoscope 109: 1334-1338, 1999

18. Teymoortash A, Hoch S, Eivazi B and Werner JA. Postoperative morbidity after different types of selective neck dissection. Laryngoscope 120: 924-929, 2010

19. Fang QG, Shi S, Zhang X, Li ZN, Liu FY and Sun CF. Upper extremity morbidity after radial forearm flap harvest: a prospective study. J Int Med Res 42: 231-235, 2014 